\title{
The 6th International Workshop on Pervasive Eye Tracking and Mobile Eye-Based Interaction
}

\author{
Diako Mardanbegi \\ Lancaster University \\ Lancaster, U.K. \\ d.mardanbegi@lancaster.ac.uk \\ Shahram Jalaliniya \\ DTU \\ Copenhagen, Denmark \\ shaja@dtu.dk
}

Mohamed Khamis

Media Informatics Group

LMU Munich

Munich, Germany

mohamed.khamis@ifi.Imu.de
Päivi Majaranta

Tampere Unit for Human

Computer Interaction

School of Information Sciences

University of Tampere

Tampere, Finland

paivi.majaranta@sis.uta.fi

Paste the appropriate copyright statement here. ACM now supports three different copyright statements: - ACM copyright: ACM holds the copyright on the work. This is the historical
approach.

License: The author(s) retain copyright, but ACM receives an exclusive

publication license.

Open Access: The author(s) wish to pay for the work to be open access. The additional fee must be paid to ACM.

This text field is large enough to hold the appropriate release statement assuming it is single spaced in a sans-serif 7 point font.

Every submission will be assigned their own unique DOI string to be included here.

\begin{abstract}
Previous work on eye tracking and eye-based human-computer interfaces mainly concentrated on making use of the eyes in traditional desktop settings. With the recent growth of interest in wearable computers, such as smartwatches, smart eyewears and low-cost mobile eye trackers, eye-based interaction techniques for mobile computing are becoming increasingly important. PETMEI 2016 focuses on the pervasive eye tracking paradigm as a trailblazer for mobile eyebased interaction to take eye tracking out into the wild, to mobile and pervasive settings. We want to stimulate and explore the creativity of these communities with respect to the implications, key research challenges, and new applications for pervasive eye tracking in ubiquitous computing The long-term goal is to create a strong interdisciplinary research community linking these fields together and to establish the workshop as the premier forum for research on pervasive eye tracking.
\end{abstract}

\section{Author Keywords}

Eye tracking; gaze-based interaction; wearable and mobile

\section{Introduction}

Eye tracking technology has considerably matured in recent years and eye-based interaction is gaining attention in various application areas. While early work focused on gaze for input in desktop settings, eye tracking is now mov- 
09.30 - 09.35 Opening

09.35 - 10.30 Keynote

10.30 - 11.00 Coffee break

11.00 - 11.15 Introductory Round

11.15 - 12.30 Presentations

12.30 - 13.30 Lunch

13.30 - 14.45 Presentations II

14.45 - 15.30 Demos

15.30 - 16.00 Coffee break

16.00 - 17.30 Discussion session

17.30 - 18.20 Plenum Discussion

18.20 - 18.30 Wrap-up

Table 1: Preliminary workshop schedule of PETMEI 2016 ing into pervasive and mobile settings. In everyday life, how and where we look provide rich information for understanding human interactions with real-world environments. Our gaze behaviors are closely related with human attention and are good indicators of visual interest. A growing number of researchers study eye-based interaction in pervasive daily-life settings, for example, eye tracking is used to assist life logging [10], pervasive display interactions [4, 11], user authentication [5] and distributed communication in mobile settings [8].

We call this new paradigm pervasive eye tracking - continuous eye monitoring and eye-based interaction 24/7 [1]

The potential applications for the capability to track and analyze eye movements anywhere and any time call for new research to further develop and understand visual behavior and eye-based interaction in mobile daily-life settings. Pervasive eye tracking opens up new research across a number of disciplines including ubiquitous computing, eyetracking research, ego- centric vision, cognitive psychology, and design. Identifying the implications and addressing challenges of pervasive eye tracking and mobile eyebased interaction require an interdisciplinary approach at the crossroads of these fields.

\section{The PETMEI workshop series}

PETMEI has been taking place annually since 2011. Except for year 2013, PETMEI always took place in conjunction with UbiComp. PETMEI 2011, 2012, 2014and 2015 attracted approximately 30 participants each, who enjoyed $11,9,9$ and 6 oral presentations respectively as well as demos of latest mobile eye trackers [6]. PETMEI 2013 [2] was held as a conference track at the $17^{\text {th }}$ European Conference on Eye Movements (ECEM 2013), in which 60 participants attended 12 oral presentations.

\section{Objectives}

The main objective of the workshop is to bring together researchers and practitioners working in $\mathrm{HCl}$, UbiComp, Eye tracking, context-aware computing, and computer vision. The workshop will create a forum for these researchers to gather, present their ideas, and to discuss techniques and applications that go beyond classical eye tracking and stationary eye-based interaction. Specifically, we want to encourage these communities to think about the implications of pervasive eye tracking for context-aware computing, i.e. the ability to track eye movements continuously for long term. The workshop aims to identify key research challenges in pervasive eye tracking and mobile eye-based interaction and to discuss the technological and algorithmic methods required to address them. The workshop shall provide intellectual stimuli and build bridges for future collaborations between these communities. The long-term goal is to create a strong interdisciplinary research community linking the different research fields and to establish the workshop as the premier forum for research on pervasive eye tracking in context-aware computing.

\section{Topics of Interest}

Topics of interest cover computational methods, new applications and use cases, as well as eye tracking technologies for pervasive eye tracking and mobile eye-based interaction.

Previous research in $\mathrm{HCl}$ and eye tracking has resulted in a large variety of methods, e.g. for eye detection and tracking [3], automated eye movement analysis [9], or evaluation of eye movement classification algorithms [7]. We invite participants to reflect on the specific characteristics of pervasive eye tracking systems and post-WIMP user interfaces and to contrast them with classical methods for eye tracking, eye movement analysis, eye-based interaction, and evaluation. We solicit contributions reporting on methodological and 


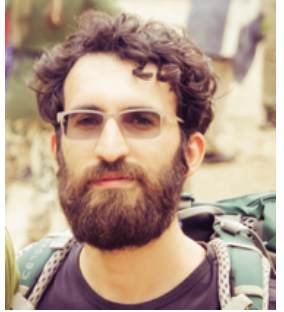

Diako Mardanbeigi is a postdoctoral researcher at the Computing and Communication department of the Lancaster University. His research interest is on mobile eye tracking, multimodal gaze interaction and ubiquitous eye tracking.

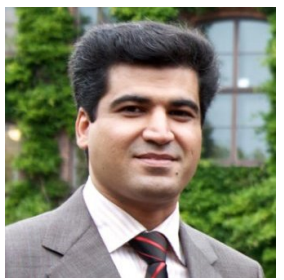

Shahram Jalaliniya is a postdoctoral researcher at the Management Engineering department of the Technical University of Denmark. His main research interest is gaze interaction on wearable computers and pervasive gaze eye-based interaction. technological advances on all components of mobile eye tracking systems. This includes computer vision techniques for face and eye detection, tracking, pupil localization, calibration, gaze estimation, pattern recognition and machine learning techniques for automated eye movement analysis, as well as methods and user studies to evaluate interaction techniques. PETMEI also covers latest advances in mobile eye tracking equipment including creative use of existing measurement techniques as well as potentially new types of tracking hardware. In summary, submissions addressing innovative pervasive eye tracking methods could cover:

- Tools for detecting, tracking, and analyzing the face, pupil, and eye movements

- Gaze-supported multimodal interaction techniques

- Methods and devices for eye Tracking on wearables (e.g. smart watches and smart glasses) and mobile devices (e.g. smart phones and tablets)

- Integration of pervasive eye tracking and contextaware computing

- Robust eye tracking for indoor/outdoor environments

In addition to contributions reporting on methodological advances we also attract submissions that explore innovative applications. By inviting researchers from different disciplines, we aim to identify particularly promising applications (the "killer applications") for pervasive eye tracking and mobile eye-based interaction.

- Pervasive eye-based interaction with displays, real and virtual environments

- Eye interaction with robots and virtual characters

- Mobile attentive user interfaces

- Eye-based activity and context recognition

- Cognition-aware systems and user interfaces

- Security \& privacy for pervasive eye-tracking systems

- Eye tracking and gaze interaction in healthcare

\section{Format}

PETMEI 2016 is a one-day workshop. We expect around 30 participants with backgrounds in eye tracking, contextaware computing, $\mathrm{HCl}$, and computer vision. As the workshop covers a new area of research at the crossroads of these fields, we aim to attract domain experts and also newcomers with an interest in the field.

\section{Before the workshop \& CFP}

The Call for Papers will be distributed in multiple research communities, including those of ubiquitous and pervasive computing, eye tracking, computer vision, and $\mathrm{HCl}$. We will set up a webpage ${ }^{1}$, and use online media to disseminate the call. We solicit submissions of up to 10 pages that describe original research related to pervasive eye tracking research. All submissions have been peer-reviewed via an international program committee. Demos of latest development of pervasive eye tracking have been encouraged from all participants. This year, we are aiming to invite the participants to provide us some information through the website about their main research interest and some of their research challenges. This will help us organize the discussion session and set topics and groups accordingly.

\section{During the workshop}

This section will be completed for the final version.

The timeline for the workshop is shown in Table 1. After the keynote, participants will briefly introduce themselves and their research interests (30 seconds each). Each paper accepted for presentation will be given 20 minutes incl. questions, which is subject to change in case of accepting more than 9 papers. In the afternoon, participants will split into groups. Each group will be asked to come up with a topic for a potential $\mathrm{CHI}$ workshop. The topic should be of their interest and within the context of PETMEI. Each group will

\footnotetext{
${ }^{1}$ http://2016.petmei.org
} 


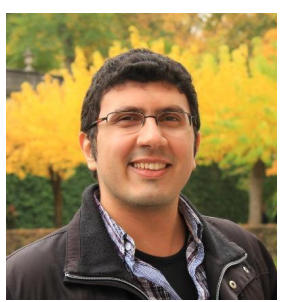

Mohamed Khamis is a PhD student at the University of Munich (LMU), Germany. His research focuses on seamless gaze-based interaction with pervasive displays by developing usable methods for interacting with public displays and mobile devices using gaze.

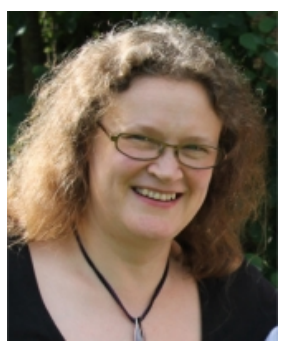

Päivi Majaranta $(\mathrm{PhD}$ in Interactive Technology) is a researcher and lecturer at University of Tampere, Finland. She gives courses on usability and interaction design. Her research interests include gaze interaction, multimodal interfaces, accessibility, $\mathrm{UX}$ and $\mathrm{HCl}$. prepare a proposal which will then be discussed among all participants in a plenary session. The best application will be awarded and could later be submitted to $\mathrm{CHI} 2017$.

\section{After the Workshop \& Publication}

Papers accepted for oral presentations will be included in the ACM Digital Library and in the supplemental UbiComp 2016 proceedings. Submissions accepted as a poster, presentation slides, and demo-related material will be made available on the workshop website.

\section{Organizers and Steering Committee}

The workshop will be organized by four researchers who are actively involved in the research fields of eye tracking, human computer interaction, and ubiquitous computing

Additionally, the PETMEI workshop series is supported by a steering committee of international eye tracking experts: Andreas Bulling, MPI Informatics, Germany

Dan Witzner Hansen, IT University of Copenhagen, Denmark

Paivi Majaranta, University of Tampere, Finland

Carlos Hitoshi Morimoto, University of São Paulo, Brasil Pernilla Qvarfordt, FX Palo Alto Laboratory, U.S.

\section{REFERENCES}

1. 2011. PETMEI '11: Proceedings of the 1st International Workshop on Pervasive Eye Tracking \& Mobile Eye-based Interaction. ACM, New York, USA.

2. Andreas Bulling and Roman Bednarik. 2013. 3rd International Workshop on Pervasive Eye Tracking and Mobile Eye-Based Interaction. In Proc. of ECEM'13.

3. Dan Witzner Hansen and Qiang Ji. 2010. In the eye of the beholder: A survey of models for eyes and gaze. Pattern Analysis and Machine Intelligence, IEEE Transactions on 32, 3 (2010), 478-500.
4. Shahram Jalaliniya and Diako Mardanbegi. 2016. Seamless interaction with scrolling contents on eyewear computers using optokinetic nystagmus eye movements. In Proc. of ETRA '16. ACM, New York, USA.

5. Mohamed Khamis, Florian Alt, Mariam Hassib Emanuel von Zezschwitz, Regina Hasholzner, and Andreas Bulling. 2016. GazeTouchPass: Multimoda Authentication Using Gaze and Touch on Mobile Devices. In Proc. CHI EA'16. ACM, New York, USA

6. Peter Kiefer, Yanxia Zhang, and Andreas Bulling. 2015. The 5th international workshop on pervasive eye tracking and mobile eye-based interaction. In Adjunct Proc. UbiComp'15. ACM, 825-828.

7. Oleg V Komogortsev, Sampath Jayarathna, Do Hyong Koh, and Sandeep Munikrishne Gowda. 2010.

Qualitative and quantitative scoring and evaluation of the eye movement classification algorithms. In Proc. ETRA'10. ACM, 65-68.

8. Diako Mardenbegi and Pernilla Qvarfordt. 2015. Creating gaze annotations in head mounted displays. In Proceedings of the 2015 ACM International Symposium on Wearable Computers. ACM, 161-162.

9. Dario D Salvucci and John R Anderson. 2001. Automated eye-movement protocol analysis. Human-Computer Interaction 16, 1 (2001), 39-86.

10. Julian Steil and Andreas Bulling. 2015. Discovery of Everyday Human Activities From Long-Term Visual Behaviour Using Topic Models. In Proc. UbiComp'15.

11. Yanxia Zhang, Ming Ki Chong, JÃürg MÃijller, Andreas Bulling, and Hans Gellersen. 2015. Eye Tracking for Public Displays in the Wild. Personal and Ubiquitous Computing 19, 5 (2015), 967-981. 